\title{
Photon absorption and photocurrent in solar cells below semiconductor bandgap due to electron photoemission from plasmonic nanoantennas
}

Novitsky, Andrey; Uskov, Alexander; Gritti, Claudia; Protsenko, I. E.; Kardynal, Beata; Lavrinenko, Andrei

Published in:

Progress in Photovoltaics

Link to article, DOI:

10.1002/pip.2278

Publication date:

2014

Document Version

Peer reviewed version

Link back to DTU Orbit

Citation (APA):

Novitsky, A., Uskov, A., Gritti, C., Protsenko, I. E., Kardynal, B., \& Lavrinenko, A. (2014). Photon absorption and photocurrent in solar cells below semiconductor bandgap due to electron photoemission from plasmonic nanoantennas. Progress in Photovoltaics, 22(4), 422-426. https://doi.org/10.1002/pip.2278

\section{General rights}

Copyright and moral rights for the publications made accessible in the public portal are retained by the authors and/or other copyright owners and it is a condition of accessing publications that users recognise and abide by the legal requirements associated with these rights.

- Users may download and print one copy of any publication from the public portal for the purpose of private study or research.

- You may not further distribute the material or use it for any profit-making activity or commercial gain

- You may freely distribute the URL identifying the publication in the public portal 


\title{
Photon absorption and photocurrent in solar cells below
}

\section{semiconductor bandgap due to electron photoemission}

\section{from plasmonic nanoantennas}

\author{
A. Novitsky, ${ }^{1, \text { a) }}$ A.V. Uskov, ${ }^{1,2,3}$ C. Gritti, ${ }^{1}$ I.E. Protsenko, ${ }^{2,3}$ B. E. Kardynał, ${ }^{1,4}$ \\ and A.V. Lavrinenko ${ }^{1}$ \\ ${ }^{1}$ DTU Fotonik, Department of Photonics Engineering, Technical University of Denmark, Ørsteds \\ Plads 343, DK-2800 Kgs. Lyngby, Denmark \\ ${ }^{2}$ P. N. Lebedev Physical Institute, Leninsky pr. 53, 117924 Moscow, Russia \\ ${ }^{3}$ Plasmonics LTD, Nijnie Polia street, 52/1, 119382, Moscow, Russia \\ ${ }^{4}$ Peter Grünberg Institute (PGI-9), Research Centre Jülich GmbH, 52425 Jülich, Germany
}

\begin{abstract}
We model the electron photoemission from metal nanoparticles into a semiconductor in a Schottky diode with a conductive oxide electrode hosting the nanoparticles. We show that plasmonic effects in the nanoparticles lead to a substantial enhancement in photoemission compared to devices with continuous metal films. Optimally designed metal nanoparticles can provide an effective mechanism for the photon absorption in the infra-red range below the semiconductor bandgap, resulting in the generation of a photocurrent in addition to the photocurrent from band-to-band absorption in a semiconductor. Such structure can form the dais of the development of plasmonic photoemission enhanced solar cells.
\end{abstract}

Plasmonic photovoltaics is a well established scientific discipline, which exploits plasmonic effects in metallic nanostructures in order to improve the efficiency and decrease the cost of solar cells (SCs), as well as to develop new concepts in photovoltaic devices [1]. Two basic,

a) Electronic mail: anov@fotonik.dtu.dk 
well-known plasmonic effects can be used to increase the photovoltaic efficiency of thin SCs: (a) resonant scattering of solar light by plasmonic nanoparticles into a thin absorbing film [1,2]; (b) increased optical absorption in the near-field zone surrounding plasmonic nanoantennas $[1,3]$. Both effects lead to the enhanced absorption of light in SC structures. In thick SCs, plasmonic nanoparticle layers can be effective as antireflection coatings [4]. A distinguishing feature of the above mechanisms is that if a semiconductor absorber of a SC absorbs the part of the solar spectrum that corresponds to photon energies $\hbar \omega>E_{g}$, where $E_{g}$ is the semiconductor bandgap energy, then the use of plasmonic nanoparticles in the SCs only results in an increase in absorption in the same part of the solar spectrum $\left(\hbar \omega>E_{g}\right)$. In such configuration only the optical properties of the plasmonic structures are exploited, and the harvesting of solar photons with $\hbar \omega<E_{g}$ is not possible.

Recently, several papers have also considered the enhanced photoemission of electrons from plasmonic nanoparticles (nanoantennas), i.e., a "plasmonic" photoemission, in order to improve the performance of solar cells. The plasmonic photoemission was proposed to realize the photoconductivity in plasmonic metamaterials [5] and increase the absorption of photons with $\hbar \omega<E_{g}$ near the bandgap energy [6]. Both effects are expected to improve the efficiency of SCs and result in the development of new photodetectors, in which nanoparticles act as active nanoantennas [7]. The photoemission of an electron from a metal into a semiconductor occurs if the photon energy $\hbar \omega$ is larger than the work function $W_{b}$ at the metal-semiconductor interface, i.e., $W_{b}<\hbar \omega$ (Fig.1a). The key idea is that a localized plasmonic resonance (LPR) in a nanoparticle can lead to a substantial increase in the absorption of photons with energies $\hbar \omega$ close to the LPR energy $\hbar \omega_{\text {LPR }}$ in the solar cell. More importantly, if $W_{b}<\hbar \omega_{L P R}<E_{g}$, then absorbed photons with energies in the range $W_{b}<\hbar \omega<E_{g}$ can induce a current of photoemitted hot electrons, which adds to the photocurrent produced 
by the absorption of photons for which $\hbar \omega>E_{g}$ in the semiconductor. It is important to note that for $W_{b}<\hbar \omega_{L P R}<E_{g}$ the absorption of light by metal nanoparticles does not affect the absorption of photons with $\hbar \omega>E_{g}$ in the semiconductor core of the SCs. The resulting total photocurrent and possibly also the photovoltaic conversion efficiency of the device are therefore higher.

In this Letter, we perform numerical study of a Schottky diode structure composed of a semiconductor/plasmonic nanoparticles/transparent conductive oxide stack. We show that the enhanced photon absorption below the semiconductor bandgap and a photocurrent as a result of the photoemission of electrons from plasmonic nanoantennas, can be achieved through the proper design of the nanoantennas (in particular the sizes and shapes of the nanoparticles). Through such additional mechanism of absorption of photons with $\hbar \omega<E_{g}$ the photovoltaic efficiency of SCs can be increased and plasmonic photoemission enhanced solar cells can be realized. In contrast to paper [7], in which the analysis of experimental results is based on semi-phenomenological Fouler's formula [8] for the photoemission, our calculations are based on the theory of the photoeffect from metallic nanoparticles $[5,9,10]$. We demonstrate promising quantum efficiency of the device.

Fig.1b shows the structure considered in the present study. It is composed of a square grid of cylindrical silver nanoparticles of height $h$ and elliptical cross-section with the semi-axes $R_{l}$ (long) and $R_{s}$ (short) deposited on a GaAs substrate. The grid has period $L$, so the nanoparticle areal density is $n_{\text {nапо }}=1 / L^{2}$. The nanoparticle layer is covered with conductive indium tin oxide (ITO). The structure is illuminated with light of wavelength $\lambda$ and intensity $S$, incident normal to the structure surface. If the energy of a hot electron generated after the absorption of a photon is higher than $0.83 \mathrm{eV}$, which is the Schottky barrier height of Ag on nGaAs, then the electron can be transferred into the semiconductor (Fig.1a). Thus, the photoemitted electron contributes to the photocurrent in the structure. The current continuity 
is maintained by recharging the nanoparticles from the ITO layer through an external circuit (not shown in Fig.1b).

We chose to study a simple Schottky barrier SC, although it had been shown [11], that metal-insulator-semiconductor (MIS) SCs have substantially better performance. They eliminate the recombination current, whereas the photocurrent flow is not affected if the insulator is thin enough to allow tunneling. This simplification does not affect the conclusions of the present simulations, while making them easier. In particular, by ignoring the insulator we can assume a constant permittivity of the semiconductor, which we expect to result in only a small shift of the LPR energy. Significantly, since the insulator is thin enough to permit the tunneling of hot photoelectrons, the cut-off wavelengths of the spectral response of both the Schottky diode and MIS solar cell are the same.

If a nanoparticle of size $a_{\text {nano }}$ is much larger than the de Broglie electron wavelength $\lambda$ in the metal, $\lambda \propto 5 \AA$ in silver, then (a) we can neglect size quantization in the nanoparticle and calculate the normal component of the photoemission current density $j_{n}$ through the nanoparticle surface into the semiconductor substrate using the theory of electron photoemission from the bulk metal through its flat boundary; (b) we can assume that the dominant mechanism for the photoemission of electrons is photon absorption by the electrons during their collisions with the surface of the metal [9]; and (c) we can assume that $j_{n}$ is proportional to the square of the electric field component $E_{n}$ inside the metal, normal to the nanoparticle metal-semiconductor interface $[5,10]$ :

$$
j_{n}=q C_{\text {emission }} \cdot\left|E_{n}\right|^{2},
$$

where coefficient $C_{\text {emission }}$ depends, in particular, on the wavelength of the light $\lambda$ and the work function $W_{b}$. The analytical expression for $C_{\text {emission }}$ for a Schottky barrier (and a 
trapezoidal tunneling barrier) can be found elsewhere $[5,12]$. Obviously, $C_{\text {emission }}$ is non zero only if $\hbar \omega>W_{b}$.

The average photocurrent density $J$ in the device is

$$
J=L^{-2} q C_{\text {emission }} \cdot \int_{A_{n s}} d s\left|E_{n}\right|^{2}=q C_{\text {emission }}\left|E_{\text {inc }}\right|^{2} \cdot \tilde{J},
$$

where the integral is taken over $A_{m s}$ - the area of the contact between the metal nanoparticle and semiconductor substrate. The quantity

$$
\tilde{J}=L^{-2}\left|E_{i n c}\right|^{-2} \cdot \int_{A_{m s}} d s\left|E_{n}\right|^{2}
$$

is a "dimensionless photocurrent density", which describes the "electromagnetic" properties of the structure (in our case the antenna properties of the plasmonic nanocylinders) relevant for photoemission, while $E_{\text {inc }}$ is the light field incident on the structure from vacuum (Fig.1b). Correspondingly, the quantum efficiency of the device $\eta=(J / q) /(S / \hbar \omega)$, where $S=c\left|E_{\text {inc }}\right|^{2} / 8 \pi$ is the intensity of the incident light, is

$$
\eta=\eta_{0} \tilde{J}, \quad \eta_{0}=8 \pi \hbar \omega C_{\text {emission }} / c .
$$

The results of numerical calculations of $\tilde{J}$ and light absorption in the structure are presented below. In simulations performed using the CST Microwave Studio [13] (the solver employs a finite integration technique for solving Maxwell's equations in integral form), we used the Drude-Lorentz formula for the complex dielectric function $\varepsilon_{m}$ of silver nanoparticles (plasma frequency $\omega_{p}=1.37 \times 10^{16} \mathrm{rad} / \mathrm{s}$ and collision frequency $\gamma=27.3 \times 10^{12} 1 / \mathrm{s}$ ). The permittivities of the ITO and GaAs layers are assumed to be frequency independent and equal to 4.67 and 12.86 , respectively $[14,15]$. For the latter material the assumption of the constant permittivity is only valid for photon energies below the absorption edge of GaAs. The constant real-valued permittivity of the ITO is chosen to get the pure effect of localized plasmons. A realistic model of the ITO only tunes positions and strengths of resonances. The 
light absorption is calculated as the part of the incident light flux that is neither reflected $(R)$ nor transmitted ( $T$ ) through the structure,i.e. $A=1-R-T$.

Fig. 2 shows absorption spectra for light polarized along the long and short axes of a nanoparticle. The absorption spectra for a circular cylindrical nanoparticle are expectedly identical for both polarizations, whereas an elliptical cylinder with $R_{l} \neq R_{s}$ has polarizationdependent plasmonic resonances that are strongly shifted from each other. By setting the long semi-axis to $R_{l}=20 \mathrm{~nm}$ and gradually decreasing the short semi-axis $R_{s}$ from 20 to 7.5 $\mathrm{nm}$, the red and blue shifts of the plasmonic resonance occur for light polarized along the long (Fig.2a) and short (Fig.2b) semi-axes, respectively. This property of the plasmonic resonances of elliptical nanoparticles can be used for the realization of a polarizationdependent electron photoemission based photodetector. In the context of the solar cell design, elliptical nanoparticles can be used to tune the plasmon resonance to a lower frequency, while maintaining the small volume of the nanoparticle, what is necessary for the efficient photoemission.

Plasmonic resonances for light polarized along the long semi-axes (Fig.2 a) of all nanoparticles considered here lie at wavelengths longer than $\lambda_{g}=0.87 \mu \mathrm{m}$, which corresponds to the GaAs bandgap $E_{g}=1.43 \mathrm{eV}$ and shorter than $1.49 \mu \mathrm{m}$, which corresponds to $W_{b}$ for the Ag-GaAs interface (Fig.2a). Therefore, the spectral responsivity of the device is extended to longer wavelengths in comparison with GaAs. Meanwhile, for light polarized along the short axes of the nanoparticles with a relatively small value of $R_{s}(<15 \mathrm{~nm})$ the photoemission occurs for photon energies $\hbar \omega>E_{g}$. Then the photon absorption due to the band-to-band transitions in GaAs may be more efficient for generating the photocurrent. It may, however, still be a useful approach to reduce the total thickness of the solar cell.

A signature of the presence of localized surface plasmons can be recognized in both the absorption, $A$, and the photocurrent, $\tilde{J}$, spectra, as shown in Fig.3 for different nanoparticle 
densities. The positions of the photocurrent maxima (Fig.3b) coincide with the positions of the absorption resonances plotted in Fig.3a. Both resonances are red-shifted and broadened with increasing the nanoparticle density (decreasing $L$ ). This behavior can be explained by an increase in interactions between nanoparticles [4]. It should be stressed again that the resonance peaks are at wavelengths between 1.1 and $1.3 \mu \mathrm{m}$, thus corresponding to energies below the semiconductor bandgap.

When the period $L$ is varied from 80 to $140 \mathrm{~nm}$ for the fixed size of the nanoparticles $\left(R_{l}=20 \mathrm{~nm}, R_{s}=7.5 \mathrm{~nm}\right)$, the peak magnitude of the plasmonic resonance passes through its maximum at $L_{\max } \approx 100 \mathrm{~nm}$ (see the inset to Fig.3a). The dimensionless photocurrent $\tilde{J}$ exhibits similar behavior as demonstrated in the inset to Fig. $3 \mathrm{~b}$. The origin of this behavior can be explained intuitively. The contribution of plasmonic nanoparticles to the absorption and photoemission must tend to zero for very low nanoparticle densities (large $L$ ). On the other hand, localized plasmon effects must vanish for a dense enough set of nanoparticles (small $L$ ), i.e. when they start bridging with each other to form a homogeneous film.

The case of a homogeneous film corresponds to a standard semiconductor-metal Schottky diode [16]. In this case, the metal is adjacent to the semiconductor at every point on the interface, and the localized plasmon effect is absent. Such device can be used as a reference for comparison with plasmonic nanoantennas devices. If light is incident normally to the reference device, the field component normal to the surface is zero, and electron photoemission is absent. For comparison, if we assume that light with intensity $S$ propagates almost parallel to the continuous metal layer in the standard device (the optimal coupling to the standard device), then the dimensionless photoemission current parameter can be estimated as $\tilde{J}_{o}=1 /\left|\varepsilon_{m}\right|^{2} \approx 2 \times 10^{-4}$. The nanoantenna-induced photoemission can be characterized by the enhancement factor $F=\tilde{J} / \tilde{J}_{o}$, which is estimated to be $\sim 10^{5}$ for the plasmonic maxima shown in Fig. 3b. The presence of the plasmonic nanoantennas results in 
a substantial enhancement in the electron photoemission (by several orders of magnitude) in comparison with the non-resonant situation. According to Ref. [5] $\eta_{o} \sim 10^{-2}-10^{-3}$. Therefore, quantum efficiency $\eta \sim 0.02-0.2$ (4) can be expected at the maxima in Fig.3b, in agreement with the value $\eta \sim 0.15$ obtained in Ref. [5].

The response of a SC to the light impinging on its surface at different angles is important for its operation under diffuse light conditions. In the geometry considered in the inset to Fig. 4 the device is illuminated with light impinging at arbitrary angle $\alpha$. The electric field, the normal to the interface and the wavevector lie in the same plane. As a result of the continuity of the normal component of the Poynting vector, the intensity of the radiation in the ITO, $I=n_{\mathrm{ITO}}|E|^{2}$, can be expressed in terms of the intensity $I_{0}$ of the incident light as $I=I_{0} \cos \alpha / \cos \theta$, where $n_{\text {ITO }}$ is the refractive index of the ITO and $\alpha$ and $\theta$ are the angles of incidence and refraction, respectively. The squared field component parallel to the interface, which excites a localized plasmon in the cylinder, has the form $\left|E_{t}\right|^{2}=|E|^{2} \cos ^{2} \theta=\left(I_{0} / n_{\mathrm{ITO}}\right) \cos \theta \cos \alpha$. Its dependence on $\alpha$ is shown by the dashed curve in Fig. 4. It is in agreement with the results of numerical simulations at the resonance wavelength $(\lambda=1176 \mathrm{~nm})$. At shorter wavelengths there is still a trace of the plasmonic effect (the black solid line in Fig. 4). In contrary, at longer wavelengths (the blue line in Fig.4) the plasmonic effect is absent. Even at large angles of incidence the photocurrent at resonance is pronounced and drops to a half of its maximum value only at the angle of approximately 60 degrees. That provides a direct confirmation of the broad-angle photon harvesting ability of the SC device.

In conclusion, we have demonstrated that plasmonic nanoparticles can be used to provide a substantial enhancement in the photoemission from a metal into a semiconductor (by several orders of magnitude), when compared with a continuous metal film. The largest photocurrent is obtained near the localized plasmonic resonances of elliptical nanoparticles arranged in a periodic array. The photoemission efficiency can be as high as $20 \%$. By the 
appropriate design of the nanoparticle shape (e.g. elliptical) the photocurrent can result from the absorption of photons with energies below the semiconductor bandgap, thus extending the spectral response range of the solar cell. The effect is preserved in the broad range of angles of incidence. Therefore, in thin solar cells this behavior can be considered as an additional mechanism for the generation of the photocurrent, leading to the realization of a new type of plasmonic photoemission enhanced solar cell.

AN and AL acknowledge partial financial support from the Danish Research Council for Technology and Production Sciences via the THzCOW project. The work of AVU at DTU was supported by the Otto Moensted Foundation.

\section{REFERENCES}

[1] Atwater HA, Polman A. Plasmonics for improved photovoltaic devices. Nature Materials 2010; 9: 205-213.

[2] Catchpole KP, Polman A. Plasmonic solar cells. Optics Express 2008; 16: 21793-21800.

[3] Rockstuhl C, Lederer F. Photon management by metallic nanodiscs in thin film solar cells. Applied Physics Letters 2009; 94: 213102.

[4] Pors A, Uskov AV, Willatzen M, Protsenko IE. Control of the input efficiency of photons into solar cells with plasmonic nanoparticles. Optics Communications 2011; 284: 22262229.

[5] Uskov AV, Protsenko IE, Rudoy VM, Dement'eva OV, Leveque G, Corbett B, O'Reilly EP. Plasmonic metamaterial as a photoconductive medium. The Second Nanotechnology International Forum (Rosnanotech-2009). Moscow, October 06-09, 2009. 
Protsenko IE, Uskov AV. Photoemission from metal nanoparticles. Physics-Uspekhi 2011, accepted for publication http://ufn.ru/en/articles/accepted/35575/. Preprint http://arxiv.org/abs/1109.1869

[6] Moulin E, Luo P, Pieters B, Sukmanowski J, Kirchhoff J, Reetz W, Müller T, Carius R, Royer F-X, Stiebig H. Photoresponse enhancement in the near infrared wavelength range of ultrathin amorphous silicon photosensitive devices by integration of silver nanoparticles. Applied Physics Letters 2009; 95: 033505.

[7] Knight MW, Sobhani H, Nordlander P, Halas NJ. Photodetection with Active Optical Antennas. Science 2011; 332: 702-704.

[8] Fowler RH. The analysis of photoelectric sensitivity curves for clean metals at various temperatures. Physical Review 1931; 38: 45-56.

[9] Tamm I, Schubin S. Zur theorie des photoeffektes an metallen. Zeischrift fur Physik 1931; 68: 97-113.

[10] Brodsky AM, Gurevich YY. Theory of electron emission from metals. Nauka: Moscow, 1973.

[11] Stirn RJ, Yeh YCM, A 15\% efficient antireflection-coated metal-oxide-semiconductor solar cell. Applied Physics Letters 1975; 27: 95-98.

Mendes MJ, Luque A, Tobías I, Martí A. Plasmonic light enhancement in the near-field of metallic nanospheroids for application in intermediate band solar cells. Applied Physics Letters 2009; 95: 071105.

[12] Schenk A, Heiser G. Modeling and simulation of tunneling through ultra-thin gate dielectrics. Journal of Applied Physics 1997; 81: 7900-7909.

[13] http://www.cst.com/

[14] West PR, Ishii S, Naik GV, Emani NK, Shalaev VM, Boltasseva A, Searching for better plasmonic materials. Laser Photonics Reviews 2010; 4: 795-808. 
[15] Aspnes DE, Kelso SM, Logan RA, Bhat R. Optical properties of $\mathrm{Al}_{\mathrm{x}} \mathrm{Ga}_{1-\mathrm{x}} \mathrm{As}$. Journal of Applied Physics 1986; 60: 754-768.

[16] Chin KK, Pan SH, Mo D, Mahowald P, Newman N, Lindau I, Spicer WE. Electronic structure and Schottky-barrier formation of Ag on n-type GaAs(110). Physical Review B 1985; 32: 918-923. 

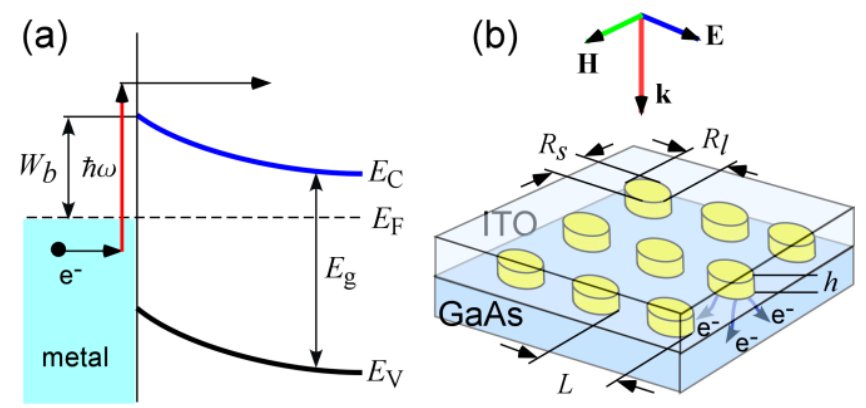

Fig. 1. (Color online) (a) Schematic diagram of the photoemission of an electron from a metal nanoparticle. An electron (black dot) absorbs a photon of energy $\hbar \omega$ during a collision with the metal-semiconductor interface, and enters the semiconductor over a Schottky barrier with the work function $W_{b}$. (b) Schematic diagram of the structure considered in the present study. Incident light induces the photoemission of electrons $\mathrm{e}^{-}$ from nanoparticles into the semiconductor.
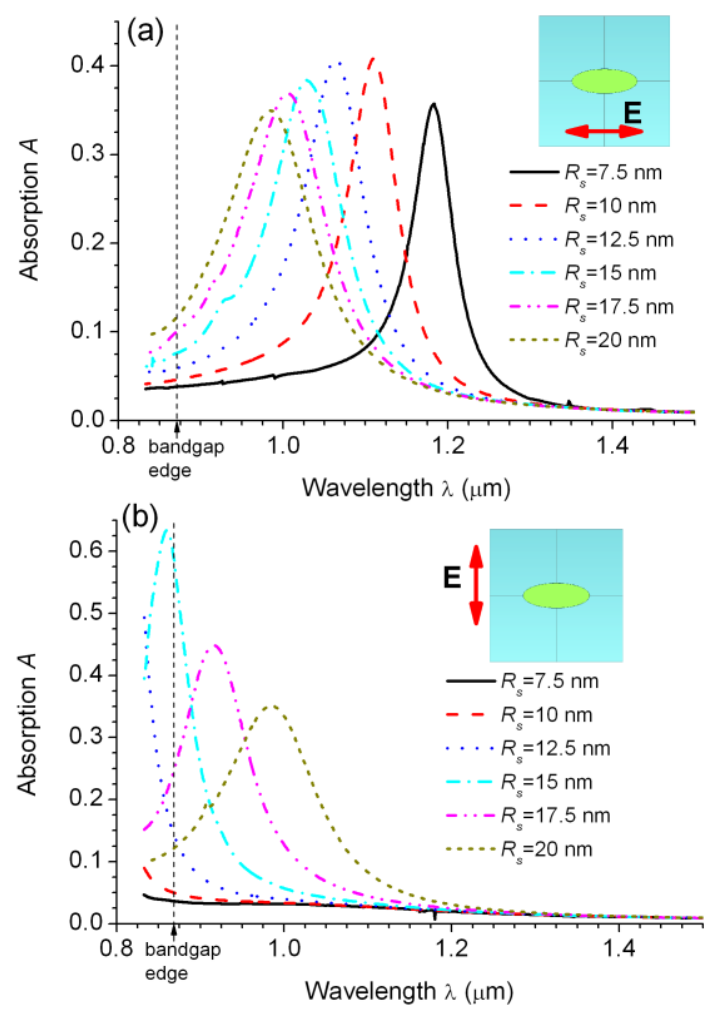

Fig. 2. (Color online) Light absorption spectra in the system of elliptic cylinders, for incident light polarized along (a) the long axes and (b) the short axes of the nanoparticles. In the calculations, $R_{l}=20 \mathrm{~nm}, h=10 \mathrm{~nm}, L=80 \mathrm{~nm}$. 

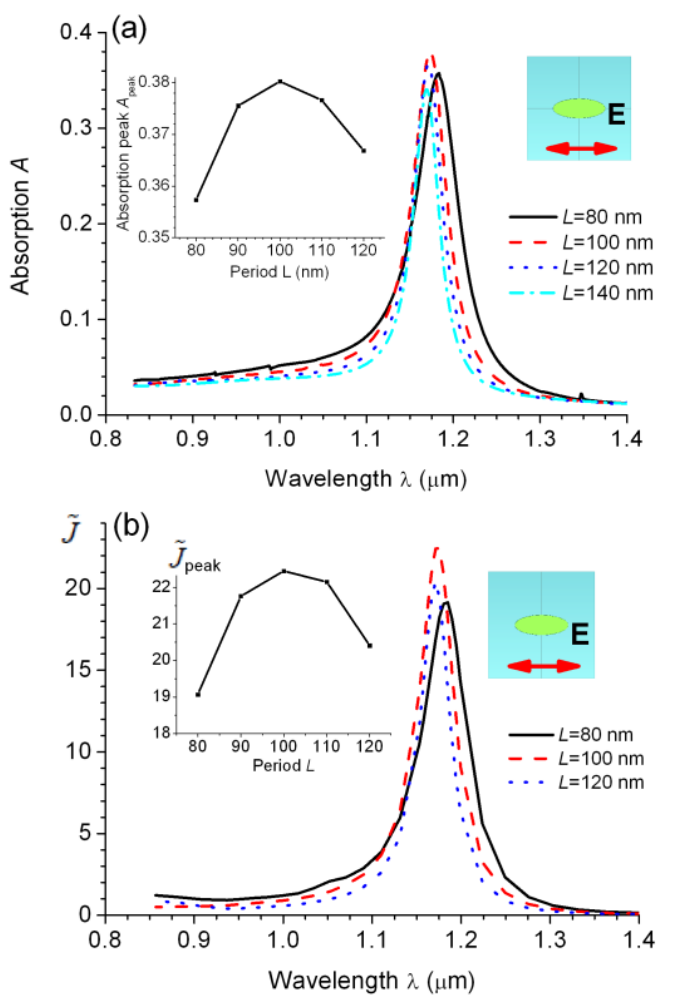

Fig. 3. (Color online) (a) Light absorption spectra and (b) spectra of the dimensionless photocurrent parameter $\tilde{J}$ for several nanoparticle densities, i.e., several values of $L$. The insets show the dependences of the resonance maxima $A_{\text {peak }}$ and $\tilde{J}_{\text {pack }}$ on $L$. In the calculations, $R_{l}=20 \mathrm{~nm}, R_{s}=7.5 \mathrm{~nm}$, and $h=10 \mathrm{~nm}$.

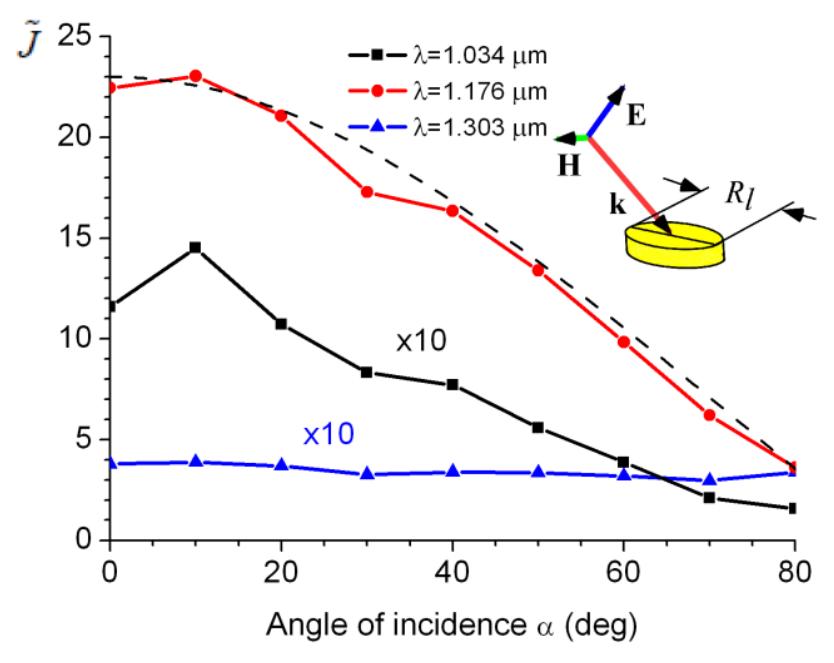

Fig. 4. (Color online) Dimensionless photocurrent $\tilde{J}$ plotted versus the light incidence angle $\alpha$, which varies in the plane of the long axes of the nanoparticles. The electric field of 
the incident wave is also in the plane of incidence. The dashed curve shows the analytical prediction. 